Abstract. Parachutes are a mass efficient way of decelerating vehicles that have entered the Martian atmosphere and are about to begin powered descent to the surface. The disk-gap-band parachute is the only parachute that has been used on actual Mars landing missions. As part of the Viking Program in 1972, this parachute was certified for deployment at Mars behind blunt bodies flying at low angles of attack, Mach numbers up to 2.2, and dynamic pressures of up to $800 \mathrm{~Pa}$. NASA is considering entry vehicle concepts for future robotic missions to Mars that would require decelerator deployment at Mach numbers of up to 3.1 and dynamic pressures of up to $1400 \mathrm{~Pa}$. These conditions are significantly outside the experience base for the disk-gap-band parachute. In addition, the decelerator may need to be deployed in an asymmetric wake. In order to fly these future Mars landing missions, an aerodynamic decelerator system needs to be developed and demonstrated in the expanded flight envelope. This paper discusses the current decelerator system used in Mars landings and the constraints imposed by it. The need for expanding these constraints and the benefits obtained are then demonstrated. Finally, a three-phase program to develop the technology needed for the expanded flight envelope is described. The end result of the technology development program will be a decelerator system that could be used for robotic missions to Mars in 2007 and beyond. In addition to Mars missions, the new decelerator technology could be used anywhere where high Mach number, low dynamic pressure deployment is required. 


\title{
Technology Development for Deployable Aerodynamic Decelerators at Mars
}

\author{
James P. Masciarelli \\ NASA Johnson Space Center, Aeroscience and Flight Mechanics Division, Houston, TX 77058 \\ 281-483-6397, james.masciarelli@jsc.nasa.gov
}

\begin{abstract}
Parachutes used for Mars landing missions are only certified for deployment at Mars behind blunt bodies flying at low angles of attack, Mach numbers up to 2.2 , and dynamic pressures of up to $800 \mathrm{~Pa}$. NASA is currently studying entry vehicle concepts for future robotic missions to Mars that would require parachutes to be deployed at higher Mach numbers and dynamic pressures. This paper demonstrates the need for expanding the parachute deployment envelope, and describes a three-phase technology development activity that has been initiated to address the need. The end result of the technology development program will be a aerodynamic decelerator system that can be deployed at Mach numbers of up to 3.1 and dynamic pressures of up to $1400 \mathrm{~Pa}$.
\end{abstract}

\section{INTRODUCTION}

Parachutes are a mass efficient way of decelerating vehicles that have entered the Martian atmosphere and are about to begin powered descent to the surface. Currently, parachutes are only certified for deployment at Mars behind blunt bodies flying at low angles of attack, within a limited range of Mach numbers and dynamic pressures. NASA is considering entry vehicle concepts for future missions that would require decelerator deployment outside of the existing constraints. Therefore, a technology development program has been initiated to expand the flight envelope of deployable aerodynamic decelerators for vehicles that land on the surface of Mars.

This paper will discuss the current decelerator system used in Mars landing missions and the constraints imposed by it. The need for expanding these constraints and the benefits obtained will then be demonstrated. Finally, a program to develop the technology needed for the expanded flight envelope will be described.

\section{Typical Mars Entry Profile}

An entry vehicle consisting of a lander, an aeroshell, and a parachute is typically used for soft landings on the surface of Mars. During atmospheric entry, the lander is contained within the aeroshell, which protects the lander from aerodynamic loads and heating as the vehicle enters the atmosphere at high velocity ( 5 to $7 \mathrm{~km} / \mathrm{s}$ at $125 \mathrm{~km}$ altitude) and decelerates. When aerodynamic loads and heating are small and the entry vehicle has descended to an altitude of 8 to $12 \mathrm{~km}$, a parachute is deployed to further slow the vehicle. The aeroshell is then separated from the lander, and the parachute helps to extract the lander from the aeroshell. The parachute then turns the lander's flight path so that it is nearly vertical and slows the lander to a terminal velocity of about 50 to $80 \mathrm{~m} / \mathrm{s}$. When the lander reaches 500 to $1000 \mathrm{~m}$ altitude, the parachute is released, and the lander uses its propulsion system to perform final maneuvers and land on the surface of Mars.

Two vehicle parameters govern the entry trajectory at a given planet: the lift-to-drag ratio (L/D), and the ballistic number. L/D is defined as the ratio of the aerodynamic lift and drag coefficients. Increasing L/D provides the entry vehicle with maneuver capability to steer out dispersions and reach a specific landing site more accurately. Ballistic number is defined as the mass of the entry vehicle divided by the product of aerodynamic drag coefficient and reference area. The ballistic number provides a measure of the ratio of inertia force to drag force. As the ballistic number increases, the entry vehicle is not slowed as much during the entry phase of flight. Therefore, when the vehicle reaches the desired parachute deployment point, it is at a higher Mach number and dynamic pressure than 
vehicles with lower ballistic numbers. In other words, the parachute deployment Mach number and dynamic pressure increase with entry vehicle ballistic number.

\section{Constraints on Parachute Deployment}

The disk-gap-band (DGB) parachute is the most widely tested at air density and Mach number combinations representative of a Mars landing, and is the only type of parachute used for actual Mars landing missions. The DGB parachute consists of a solid flat disk canopy to which has been added an open slot and an extended skirt. The DGB parachute was first certified for interplanetary use during the Viking Program, which placed two landers on Mars in 1976. At that time, a parachute development and testing program was pursued to demonstrate parachute deployment at the expected conditions and also to verify parachute performance over the entire parachute flight regime. The parachute development and test program began with wind tunnel testing, followed by low altitude aerial testing, and finally high altitude testing. The first wind tunnel tests occurred in February 1971, and the last high altitude test was performed in August 1972. Adjustments were made to the parachute system as the development and testing program progressed. A detailed account of the Viking parachute development program can be found in the open literature. Steinberg, et. al. (1973) describe the wind tunnel testing, Murrow, et. al. (1973) cover the low altitude testing, Raper, et. al. (1973) discuss the high altitude balloon launch testing process, and Moog, et. al. (1973) explain the high altitude test vehicle, test conditions, and test results.

The development work completed for the Viking deceleration system led to the DGB parachute being certified for deployment behind a blunt body flying at low angle of attack, for Mach numbers of 1.4 to 2.2, and dynamic pressures of 400 to $800 \mathrm{~Pa}$. There has been no significant work to expand this deployment envelope since that time. Consequently, all of the Mars lander missions since the Viking Program (e.g., Mars Pathfinder, Mars Polar Lander) have used DGB parachutes and tailored the entry trajectories so that the parachute could be deployed within the Mach number, dynamic pressure, and forebody wake conditions certified for the Viking lander.

\section{FUTURE MISSION REQUIREMENTS}

Future missions to Mars will require the ability to land accurately near preselected landing sites. In addition, the mass delivered to the surface is continuing to increase over that of past missions. The relationship between precision landing, increasing lander mass, and constraints on parachute deployment are now discussed, along with their affects on entry trajectory performance.

\section{Precision Landing and Increasing Entry Mass}

In past Mars landing missions, a ballistic, unguided entry trajectory was used. Ballistic entry trajectories typically fly at angles of attack near zero, generating as much drag as possible to slow the vehicle during entry, and can deliver the lander to within about $200 \mathrm{~km}$ of a target landing site. NASA is planning on using guided entry trajectories for future Mars landing missions in order to deliver a lander to within 5 to $10 \mathrm{~km}$ of a specific landing site. Guided entry trajectories make use of lift generated by the aeroshell during entry to steer to a target latitude and longitude. In order to reach the delivery accuracy of 5 to $10 \mathrm{~km}$, the entry vehicle must have a L/D of at least 0.22 , which is higher than that of past Mars entry vehicles.

The increase in entry vehicle L/D is typically accompanied by a decrease in drag. Flying at higher angles of attack and streamlining the aeroshell shape are two means of providing the necessary lift capability. A variety of aeroshell shapes are being considered that provide the L/D required for precision landing. These include variations of the Viking style sphere cone (blunt bodies) that provide L/D of up to about 0.25 , and slender bodies with L/D of up to about 0.35 . All of these shapes have lower drag for the higher L/D, therefore the entry vehicle ballistic number is increasing with $\mathrm{L} / \mathrm{D}$.

In addition to increasing the L/D of entry vehicles, future missions being studied have higher masses at entry than those of the past. Entry masses for past Mars landers (Viking, Pathfinder, and Mars Polar Lander) were in the range of 500 to $1000 \mathrm{~kg}$. However, entry masses for planned missions to Mars in 2007 and beyond are in the range of 
1800 to $3500 \mathrm{~kg}$. Conceptual designs of human missions to Mars require entry masses on the order of $60 \mathrm{mt}$. This increasing trend in entry mass also leads to an increasing trend in entry vehicle ballistic number.

\section{Entry Guidance Description}

As has been mentioned, future missions will use a guided entry trajectory to increase landing accuracy. The guidance algorithm being studied for use on future missions is the Entry Terminal Point Controller (ETPC) algorithm (Carman, et. al., 1998). The ETPC guidance algorithm uses aerodynamic lift to control the atmospheric flight path towards a specified target at parachute deployment. The guidance algorithm determines if modifications to the current atmospheric flight path are required and directs the control system to make attitude adjustments based on the navigation system input. This system modulates the vehicle bank angle such that the vehicle maneuvers towards a trajectory that will satisfy the desired final conditions. In this manner, the vehicle can accommodate offnominal entry-state or atmospheric flight conditions and achieve a significant increase in landing accuracy.

Once the guidance system has steered the entry vehicle to the desired target, the parachute system is deployed and the terminal descent phase of flight is initiated. In the ideal case, the parachute would be deployed as soon as the guidance algorithm determines that the entry vehicle has reached the target. However, because there are constraints on parachute deployment conditions, the guidance algorithm first checks if the Mach number and dynamic pressure are below the maximum limits. If the vehicle is outside of either of theses constraints, parachute deployment is delayed so that the velocity can be further reduced by aeroshell drag, until the constraint is satisfied. In this case, the vehicle flies past the target, which results in a larger final position error relative to the target than if the decelerator were deployed at the desired point. As has been mentioned, Mach number and dynamic pressure at parachute deployment increase with ballistic number. Therefore, as the ballistic number increases, the parachute deployment constraints begin to cause late parachute deployment, reducing the achievable landing accuracy.

In addition to delaying parachute deployment, the guidance system may deploy the parachute early if the vehicle falls below a minimum altitude. This logic is required in order to maintain adequate altitude margin for deceleration on the parachute and maneuvering during powered descent. If the vehicle falls below the altitude that causes the parachute to be deployed, and the vehicle is also outside of the parachute deployment constraints at this time, the parachute system may not deploy correctly or could be severely damaged, resulting in loss of the mission. Higher ballistic number vehicles tend to descend through the atmosphere more rapidly and reach the target at lower altitudes. Therefore, as the ballistic number increases, the chances for early parachute deployment increase, which results in reduced accuracy and increased probability of failed parachute deployment.

\section{Entry Performance for Two Different Ballistic Numbers}

Monte Carlo trajectory simulations were run in order to demonstrate the effect of ballistic number on parachute deployment conditions, and also to show the effect of expanding the parachute deployment envelope. The simulations include random dispersions on delivery and knowledge entry states, aerodynamic coefficients, trim angle of attack (affects L/D and ballistic number), and vehicle mass properties, as well as the Mars Global Reference Atmosphere Model (Mars GRAM), which provides variation in atmosphere properties. The entry guidance algorithm is also included, and attempts to steer the vehicle to the desired target in the presence of these disturbances.

Figure 1 shows the Monte Carlo simulation results for a $3000 \mathrm{~kg}$ entry vehicle with L/D of 0.33 and ballistic number of $146 \mathrm{~kg} / \mathrm{m}^{2}$. In this case, the Viking certified parachute deployment envelope was assumed, with maximum Mach number of 2.2 and maximum dynamic pressure of $800 \mathrm{~Pa}$. It can be seen that many cases required late parachute deployment in order to wait for the constraint on Mach number to be satisfied. It can also be seen that several cases fell below the minimum altitude of $6 \mathrm{~km}$, resulting in early parachute deployment. The final error in navigated range to the target is less than $3 \mathrm{~km}$, which is driven by exceeding the parachute deployment constraints. The error in actual range to target is $5 \mathrm{~km}$, due to both the navigation error and exceeding the parachute deployment constraints. Based on this data, $3000 \mathrm{~kg}$ is close to the maximum entry mass for this vehicle in order to maintain landing accuracy on the order of $5 \mathrm{~km}$. 

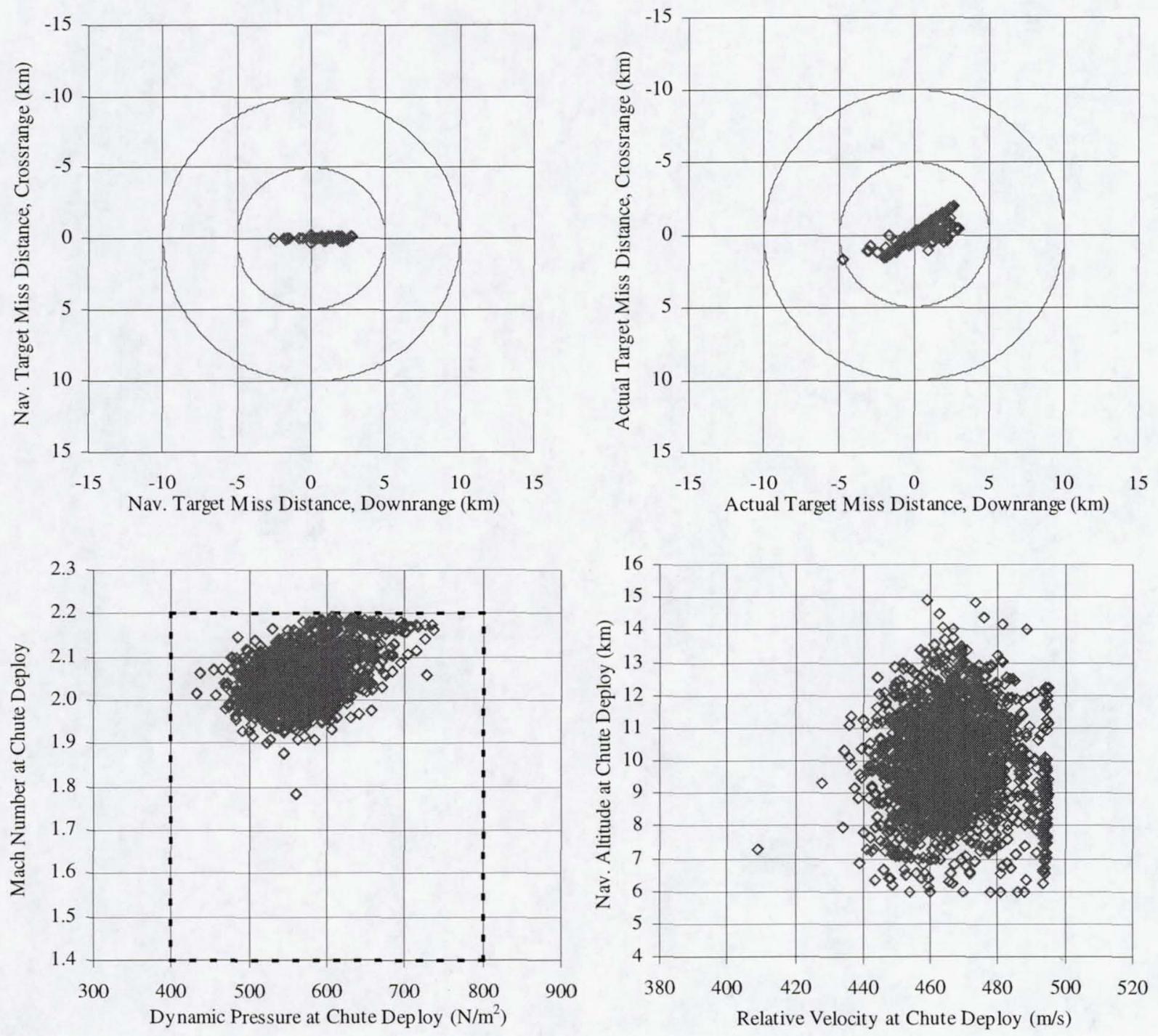

FIGURE 1. Monte Carlo Results for Entry Ballistic Number of $146 \mathrm{~kg} / \mathrm{m}^{2}$ (2000 cases).

Figure 2 shows the Monte Carlo simulation results for a $4500 \mathrm{~kg}$ entry vehicle with $\mathrm{L} / \mathrm{D}$ of 0.33 and ballistic number of $220 \mathrm{~kg} / \mathrm{m}^{2}$. In this case, an expanded parachute deployment envelope was assumed with maximum Mach number of 3.0, and maximum dynamic pressure of $1300 \mathrm{~Pa}$. It can be seen that for this case, there are essentially no late parachute deployments to satisfy the constraints on Mach number or dynamic pressure. As a result, the landing accuracy is significantly increased. As can be seen, the final error in navigated range to the target is less than $1 \mathrm{~km}$. The error in actual range to target is essentially equal to the accuracy of the navigation system.

These results show that by expanding the parachute deployment constraints to a Mach number of 3.0 and dynamic pressure of $1300 \mathrm{~Pa}$, the maximum entry vehicle mass can be increased from $3000 \mathrm{~kg}$ to $4500 \mathrm{~kg}$. This increase in entry mass would provide about a 75 percent increase in landed mass. In addition, the landing accuracy is increased to the limit of the navigation system. 

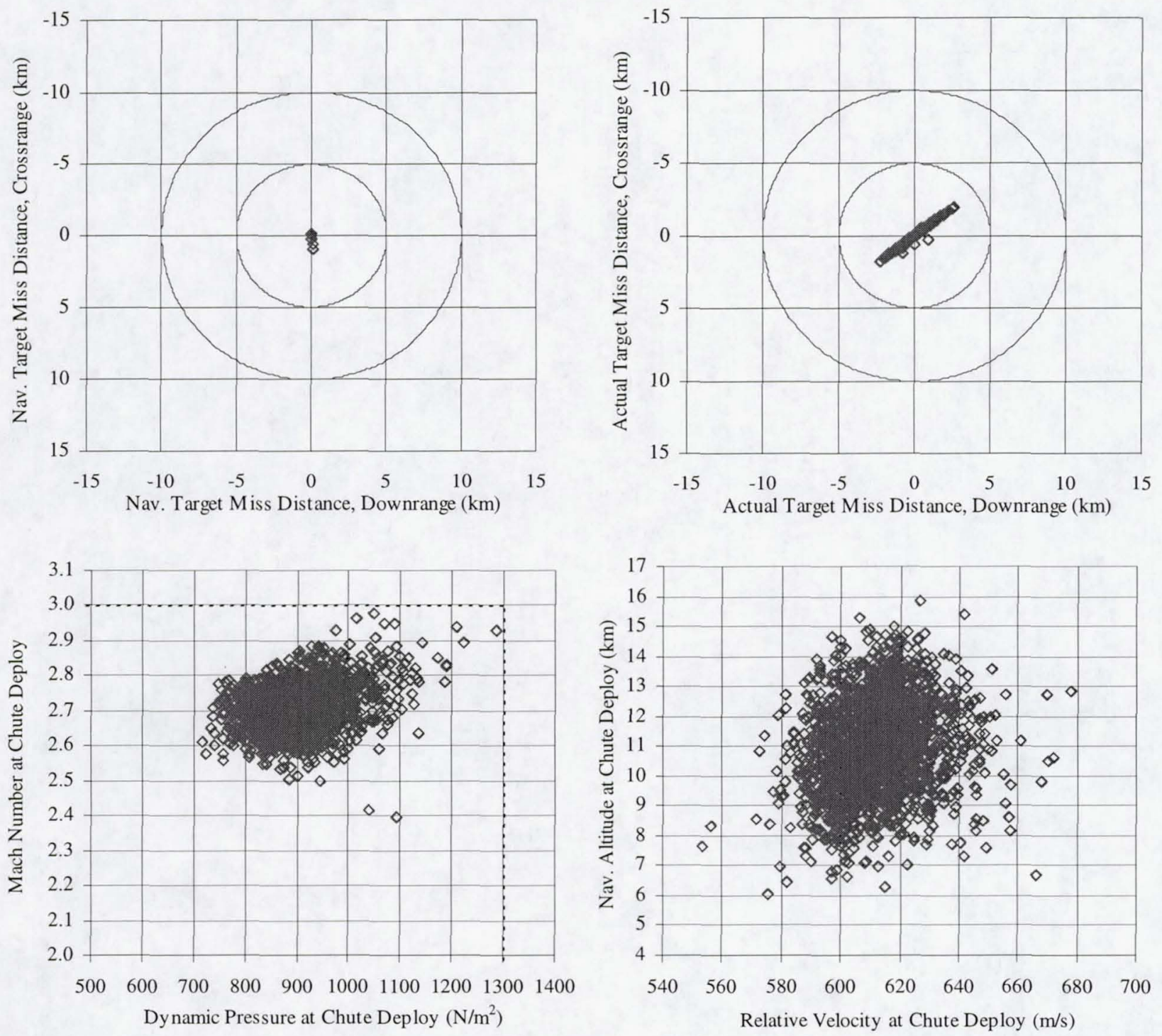

FIGURE 2. Monte Carlo Results for Entry Ballistic Number of $220 \mathrm{~kg} / \mathrm{m}^{2}$ and Expanded Deployment Envelope (2000 cases).

\section{Forebody Wake Effect on Parachute Deployment}

In addition to the higher Mach numbers and dynamic pressures at deployment, there is another problem in using the existing DGB parachute for the higher L/D entry vehicles being considered for future Mars missions. In past missions, the parachutes were deployed behind blunt body vehicles flying at low angles of attack. In this case the wake behind the entry vehicle is fairly symmetric. As has been mentioned, one way of increasing lift during entry is by flying at higher angles of attack. Some entry vehicle concepts currently being considered require trim tabs or other devices to cause the vehicle to trim at the desired angle of attack, and for other aeroshell shapes, the natural trim angle of attack could be up to 55 degrees. In either case, the wake behind the entry vehicle will not be as symmetric as it has been in past missions. Since DGB parachutes for Mars entry vehicles have only been used behind vehicles with fairly symmetric wakes, the performance and the deployment in an asymmetric wake is unknown and must be investigated. 


\section{Requirement for Expanded Parachute Deployment Envelope}

Expanding the parachute deployment constraints will enable future Mars landing missions to accurately deliver appreciably more payload mass to the surface. For some mission concepts being considered, parachute deployment is predicted to occur at Mach numbers up to 3.1 and dynamic pressures of up to $1400 \mathrm{~Pa}$. These conditions are significantly outside the experience base for the DGB parachute at Mars. In addition, the decelerator may need to be deployed in an asymmetric wake. In order to perform these contemplated missions, an aerodynamic decelerator system needs to be developed and demonstrated in this expanded flight envelope. Although it is beneficial for the decelerator system to have heritage with past missions, the system used on the future missions may not necessarily be the Viking heritage DGB parachute. In any case, a testing program similar to what was performed during the Viking Program will need to be completed before any decelerator system is used on an actual mission in the new flight conditions.

\section{AERODYNAMIC DECELERATOR DEVELOPMENT PLAN}

A three-phase technology development activity has been initiated to expand the parachute deployment envelope to Mach numbers up to 3.1 and dynamic pressures up to $1400 \mathrm{~Pa}$. During the first phase, several decelerator system design concepts that have the potential of satisfing these requirements will be developed, as well as a detailed plan for testing the concepts in a wind tunnel. The most promissing configurations will then be selected for further development. In the second phase, scale models of the selected decelerator systems will be designed, fabricated, and tested in a wind tunnel to gather basic design information. In the third phase, a single decelerator system configuration will be selected, and flight testing at high altitude will be performed. The work is expected to be completed over a period of three to four years. The following sections of this paper describe the plan in more detail.

\section{Analysis, Trade Studies, Decelerator System Design}

During the first phase of the work, analytical models will be developed and analysis and trade studies will be performed to develop candidate decelerator system design concepts. Trajectory analysis and trade studies will be performed to determine all necessary requirements for sizing the deceleration system. This will include determination of decelerator system aerodynamic coefficients and drag efficiency when combined with the entry vehicle aeroshell, assessment of aerodynamic and inertial loads, aerodynamic heating, deployment sequence and time line, and evaluation of deployment forces. Nominal and off-nominal conditions will be examined and accounted for. Off-nominal conditions will include atmosphere density variations, variation in predicted decelerator aerodynamic coefficients, variation in deceleration system mass, and variation of entry trajectory parameters. Other significant unknown quantities will be identified and analyzed to assess the impact of variation in those parameters as well.

The product of the analysis will be one or more deceleration system design concepts to meet all requirements and performance specifications identified. Designs will include definition of configurations and geometry, description of operation, materials selection, stress analysis, and weight and volume calculations. Configurations to be pursued for further evaluation in wind tunnel tests will be identified.

Once promising configurations are defined, a wind tunnel test program will be designed to evaluate the decelerator system configurations. The main objective of the wind tunnel tests is to deploy various decelerator system configurations in the expected flight conditions, study the response to changes in design parameters, and collect basic data necessary for future full-scale development. Analysis to be completed in developing the wind tunnel test plan will include definition of the scale of the models to be tested, the test conditions, test sequence, number of runs, the data to be collected, and how the data obtained from the scale model tests will be extrapolated and used for full scale design.

\section{Wind Tunnel Testing}

Wind tunnel testing of deployable aerodynamic decelerators, and parachutes in particular, is difficult. Scale models of the decelerators must be constructed so that they match the porosity and stiffness of the full-scale design as 
closely as possible. Tape, stitching, lines, and other fabric components typically have minimum sizes and cannot be scaled properly. Therefore the design of the wind tunnel model will be different than the full-scale design. With different designs, the job of interpreting the wind tunnel test results and applying them to full-scale design is made more complex. For this reason, it is desirable to use the largest scale possible for the wind tunnel models.

The wind tunnel must be large enough to prevent erroneous data due to blockage and scaling effects. Therefore it is desirable to use as large a tunnel as possible. The largest wind tunnels available for supersonic testing are the Abe Silverstein Supersonic Wind Tunnel, located at the NASA Glenn Research Center, and the 16S wind tunnel at the Arnold Engineering Development Center. The Abe Silverstein tunnel has a $3.048 \mathrm{~m}(10 \mathrm{ft})$ cross section and can reach test section speeds ranging from Mach 2.0 to 3.5. The $16 \mathrm{~S}$ tunnel has a $4.877 \mathrm{~m}(16 \mathrm{ft})$ cross section, and was originally designed to provide Mach numbers of 1.5 to 4.75 . However, as of this writing, the $16 \mathrm{~S}$ tunnel would require some refurbishment in order to reach the desired test Mach numbers. Large supersonic wind tunnels are expensive to conduct tests in. Therefore, during the wind tunnel planning phase, an assessment of the best wind tunnel facility to be used for testing will be made. Decelerator system concepts that could be tested in smaller wind tunnels would have an advantage over those that require large tunnels.

During the second phase of the development work, scale models and other decelerator system related hardware required to perform the wind tunnel tests will be designed and manufactured. This will include the entry vehicle model, the decelerator model, the decelerator deployment system for use in the wind tunnel, any decelerator system consumables, and associated mounting structure. Wind tunnel tests will be performed with Mach numbers of 2.2 to 3.1 and dynamic pressures of 800 to $1400 \mathrm{~Pa}$. Post-test analysis and data reduction will be performed, and adjustments to be made to decelerator system design for the high altitude tests will be identified.

\section{High Altitude Testing}

Tests at high altitude and velocity in order to match the Mach number and dynamic pressure expected at Mars are required before the decelerator system will be used for space flight. The high altitude tests will be designed to study the transient response of the integrated forebody, decelerator, and deployment system. This will provide an understanding of decelerator inflation time, stability, and its interaction with the forebody in a decelerating environment with inertial effects. It is desirable to test full-scale designs, however, smaller scale test articles may be used if it is appropriate.

At least four test flights are anticipated to cover the extremes of Mach number and dynamic pressure. Representative test conditions are shown in Table 1. In these tests, a forebody representative of the entry vehicle and the decelerator system will be launched to the test altitude and velocity by either a high altitude balloon plus a kick stage, or by a sounding rocket. High altitude balloons were used in the Viking certification program and offer the possibility of launching full-scale test articles. Sounding rockets would be a simpler and less costly method of launching the test articles, but their payload capability is less than that of balloons, so subscale versions would most likely be used. The decision as to which approach will be taken will be made during the initial period of the high altitude test planning.

TABLE 1. Representative High Altitude Flight Test Conditions at Earth.

\begin{tabular}{cccc}
\hline Mach & Dynamic Pressure $(\mathbf{P a})$ & Velocity $(\mathrm{m} / \mathbf{s})$ & Altitude $(\mathbf{k m})$ \\
\hline 2.2 & 1400 & 688 & 37.1 \\
2.2 & 800 & 704 & 41.2 \\
3.1 & 1400 & 996 & 42.2 \\
3.1 & 800 & 1020 & 46.5 \\
\hline
\end{tabular}

Initial effort will be spent on identifying issues related to the decelerator that will be tested, identifying overall test objectives and goals, and selecting test conditions and number of test flights required. Test vehicle decelerator system components will be developed, as well as overall test vehicle concepts with mass, power, volume, and cost 
estimates. Trajectory analysis and trade studies will be performed to develop test flight profiles. All of the analysis will be integrated into a high altitude test plan.

After the test plan is completed, the test vehicles, decelerator, and deployment systems will be designed and manufactured. Test flights could occur at about one month intervals. After each test flight is completed, post-flight analysis and data reduction will be performed in order to assess the performance of the decelerator and deployment system. Once the test flights are satisfactorily completed, the decelerator system will be nearly ready for use on actual Mars landing missions. Additional qualification testing, (e.g., environmental testing, low altitude drop tests) would be completed as part of the specific mission's development work.

\section{SUMMARY}

This paper has described how deployable aerodynamic decelerators are typically used for Mars soft-landing missions. The parachute systems being used today have constraints on deployment Mach number and dynamic pressure, which were certified during the Viking program nearly 30 years ago. Future space exploration missions will eventually require an expanded deployment envelope. The need for the expanded envelope is primary driven by more massive payloads and by higher L/D required for precision landing. The benefits of expanding the deployment envelope are an increase in payload mass, increase in landing accuracy, and increase in robustness for future space exploration missions.

Initial steps are being taken to develop the technology for aerodynamic decelerators that can be deployed at Mach numbers from 2.2 to 3.1 and dynamic pressures of 800 to $1400 \mathrm{~Pa}$. The plan begins with analytical development of decelerator system concepts that meet the requirements imposed by the expanded flight envelope. The best concepts will be tested in wind tunnels. Finally, high altitude flight testing will be carried out to fully demonstrate decelerator system performance in the expanded flight envelope. The end result will be a decelerator system that could be used for missions to Mars in 2007 and beyond. In addition to Mars missions, the new decelerator technology could be used anywhere where high Mach number, low dynamic pressure deployment is required.

\section{ACKNOWLEDGMENTS}

The author would like to acknowledge Gilbert Carman of the NASA Johnson Space Center who generated the Monte Carlo simulation results for entry vehicles with different ballistic numbers and parachute deployment constraints. The author would also like to thank Butch Cockrell and Claude Graves of the NASA Johnson Space Center for reviewing drafts of this paper and providing many useful comments.

\section{REFERENCES}

Carman, G., Ives, D., Geller, D., “Apollo-Derived Mars Precision Lander Guidance,” in A Collection of the AlAA Atmospheric Flight Mechanics Conference Technical Papers, 10-12 August 1998, Boston, MA, American Institute of Aeronautics and Astronautics, Reston, VA, 1998, Paper 98-4570.

Moog, R.D., Bendura, R.J., Timmons, J.D., Lau, R.A., "Qualification Flight Tests of the Viking Decelerator System,” in AIAA 4th Aerodynamic Deceleration Systems Conference, Palm Springs, CA, 21-23 May, 1973, American Institute of Aeronautics and Astronautics, Reston, VA, 1973, Paper 73-457.

Murrow, H.N., Eckstrom, C.V, Henke, D.W., "Development Flight Tests of the Viking Deceleration System," in AIAA 4th Aerodynamic Deceleration Systems Conference, Palm Springs, CA, 21-23 May, 1973, American Institute of Aeronautics and Astronautics, Reston, VA, 1973, Paper 73-455.

Raper, J.L., Lundstrom, R.R, Michel, F.C., "The Viking Parachute Qualification Test Technique," in AIAA 4th Aerodynamic Deceleration Systems Conference, Palm Springs, CA, 21-23 May, 1973, American Institute of Aeronautics and Astronautics, Reston, VA, 1973, Paper 73-456.

Steinberg, S., Siemers, P.M., Slayman, R.G., "Development of the Viking Parachute Configuration by Wind Tunnel Investigation," in AIAA 4th Aerodynamic Deceleration Systems Conference, Palm Springs, CA,21-23 May, 1973, American Institute of Aeronautics and Astronautics, Reston, VA, 1973, Paper 73-454. 